Jones and Toklu. HCA Healthcare Journal of Medicine (2020) 1:4

https://doi.org/10.36518/2689-0216.1112

\title{
Editorial
}

\section{Cosmetovigilance: A New Concept for the Residency Curriculum}

Jennifer Jones, MD, ${ }^{1,2}$ Hale Z Toklu, PhD $^{2,3}$

\section{Abstract}

Author affiliations are listed
at the end of this article.
Correspondence to:
Jennifer Jones, MD
Assoc. Program Director of
Family Medicine
University of Central
Florida/HCA Healthcare
(purdiejonesmd@gmail.
com)
Hale Z. Toklu, PhD
Director of Research,
HCA North Florida Division
Assoc. Prof., University of
Central Florida College of
Medicine, Dept. of Clinical
Sciences
(hale.toklu@hcahealthcare.
com)

\section{Description}

Primary care physicians need to incorporate "cosmetovigilance" into their post-graduate curriculum to increase their residents' awareness of the adverse reactions induced by the use of cosmetic products, as well as promoting good reporting behavior.

\section{Keywords}

cosmetics, adverse effects; consumer product safety; pharmacovigilance; education, medical, graduate; cosmetovigilance; curriculum; graduate medical education

While delivering patient care, assuring the safe use of medications is essential for good clinical practice. However, the concept of patient safety is not limited to medications. In recent years, new terms have been introduced to extend the concept of the safe use of herbal medicine (phytovigilance, herbavigilance) and cosmetics (cosmetovigilance).

As defined by the World Health Organization (WHO), "pharmacovigilance" is the sum of the activities related to the collection, detection, assessment, monitoring and prevention of adverse reactions occurring with medications. ${ }^{2}$ As mentioned above, the spectrum of "-vigilance" has been extended to address the safe use of herbal products and also cosmetic products. ${ }^{3}$ These new concepts are important to public health. We recently published a systematic review on this topic, which highlights the difference between the regulations in Europe, the U.S., Canada and other regions. The misidentification and underreporting of cosmetic-related adverse effects is a worldwide issue.

Even though cosmetic products are usually well-tolerated, the undesirable side effects seen with medicine can also be seen with cosmetics and toiletries. Adverse event reports are important to initiate a signal for potential risks. Furthermore, validation, assessment of causality, frequency and severity, and benefits and risks are important since they may result in eventual changes to product information, safety alerts or recalls.' However, identifying these effects is challenging due to the limited information available as well as the need to validate the reports. ${ }^{3}$ While the absence of well-established cosmetovigilance systems is a limitation, the other problem is underreporting by health professionals. There have been reports that the adverse effects of cosmetics and toiletries are underestimated, even when consumers seek medical consultation. ${ }^{4}$ On the other hand, the quantity and the quality of

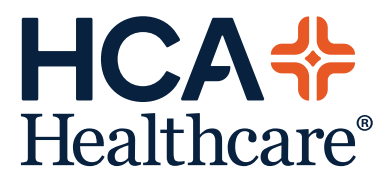

www.hcahealthcarejournal.com

(C) 2020 HCA Physician Services, Inc. d/b/a Emerald Medical Education
HCA Healthcare Journal of Medicine 


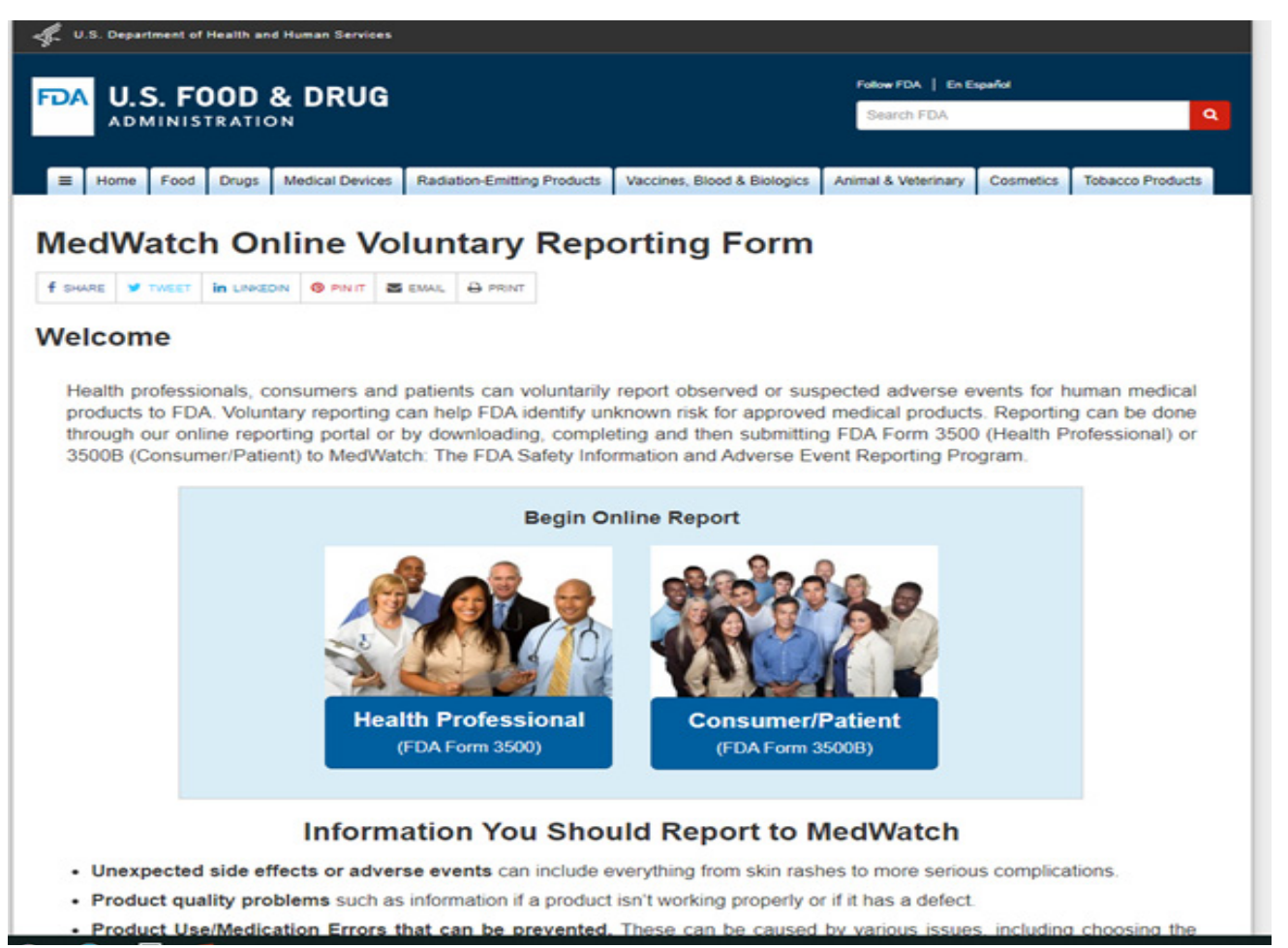

Figure 1. U.S. FDA webpage for submitting Adverse Event Reports (screenshot accessed on 3/10/2020 at https://www.accessdata.fda.gov/scripts/medwatch/index.cfm)

One of the methods below can be used to submit voluntary adverse event reports to the FDA:

1. Report Online via the MedWatch website;

2. Call the FDA at 1-800-FDA-1088 to report by telephone;

3. Fill the Reporting Form FDA 3500 and send by fax or mail.

reports can be improved by several methods, which all involve training. ${ }^{5}$

In the U.S., adverse effects are reported to the U.S. Food and Drug Administration (FDA) MedWatch System. ${ }^{6}$ (Figure 1) However, this novel concept is unfamiliar to most health care professionals, including physicians. With this evolving area of patient safety, the need to integrate cosmetovigilance into the residency curriculum has emerged. More specifically, primary care physicians must incorporate cosmetovigilance into their curriculum to increase residents' awareness of adverse reactions induced by the use of cosmetic products, as well as to promote good reporting behavior.

Effective curriculum development requires a determination of both content and implementation. ${ }^{7}$ While it is important to integrate the basics of cosmetovigilance into the curriculum, the curriculum still needs to cover the current policies and practices of the country.
Cosmetovigilance curriculum content needs to include clinical education on the identification of potential cosmetic adverse reactions. The ambulatory setting is best suited for pattern recognition of the primarily dermatologic adverse effects associated with cosmetic use. Within the current Family Medicine Residency curriculum, there are two opportunities for this to be accomplished: 1) the dermatology rotation and 2) the continuity ambulatory practice. Brief didactics highlighting common cosmetic adverse events may accompany the clinical educational experience. Additionally, formal education on the use of the FDA MedWatch System should be required and implemented through the research department. Awareness of this valuable reporting system is the key to having a bigger impact on a national platform. Filling this gap in current family medicine graduate training will serve the long-term purpose of improving the recognition of the adverse effects of cosmetic products and also help to improve the regulation of this market in the future. 


\section{Conflicts of Interest}

The authors declare they have no conflicts of interest.

The authors are employees of HCA Healthcare Graduate Medical Education, an organization affiliated with the journal's publisher.

This research was supported (in whole or in part) by HCA Healthcare and/or an HCA Healthcare affiliated entity. The views expressed in this publication represent those of the author(s) and do not necessarily represent the official views of HCA Healthcare or any of its affiliated entities.

\section{Author Affiliations}

1. Department of Family Medicine, University of Central Florida

2. HCA Healthcare Graduate Medical Education

3. Department of Clinical Sciences, University of Central Florida College of Medicine

\section{References}

1. Toklu HZ, Antigua A, Lewis V, Reynolds M, Jones J. Cosmetovigilance: A review of the current literature. J Family Med Prim Care. 2019 May;8(5):1540-1545. https://doi.org/10.4103/ jfmpc.jfmpc 44718

2. World Health Organization. Safety monitoring of medicinal products: reporting system for the general public. Geneva, Switzerland: World Health Organization; 2012. https://www.who.int/ medicines/areas/quality safety/safety efficacy/ gas safetymonitoringmp/en/

3. Vigan M, Castelain F. Cosmetovigilance: definition, regulation and use "in practice". Eur J Dermatol. 2014;24(6):643-9. https://doi.org/10.1684/ ejd.2014.2493

4. Lindberg M, Tammela M, Bostrom A, Fischer T, Inerot A, Sundberg K, et al. Are adverse skin reactions to cosmetics underestimated in the clinical assessment of contact dermatitis? A prospective study among 1075 patients attending Swedish patch test clinics. Acta Derm Venereol. 2004;84(4):291-5. https://doi. org/10.1080/00015550410025921

5. Berne B, Tammela M, Farm G, Inerot A, Lindberg $M$. Can the reporting of adverse skin reactions to cosmetics be improved? A prospective clinical study using a structured protocol. Contact Dermatitis. 2008;58(4):223-7. https://doi.org/10.1111/ j.1600-0536.2007.01309.x

6. United States Food and Drug Administration. Using Adverse Event Reports to Monitor Cos- metic Safety. https://www.fda.gov/cosmetics/ how-report-cosmetic-related-complaint/using-adverse-event-reports-monitor-cosmetic-safety Accessed on Aug 20, 2020.

7. Schneiderhan J, Guetterman TC, Dobson ML. Curriculum development: a how to primer. Fam Med Community Health. 2019;7:e000046. https://doi.org/10.1136/fmch-2018-000046 